\title{
RFID Painting Demonstration
}

\author{
Olivier Haberman ${ }^{1}$, Romain Pellerin ${ }^{2}$, Eric Gressier-Soudan ${ }^{2}$, and Ugo Haberman ${ }^{3}$ \\ ${ }^{1} 17$ rue Casimir Perier, 75007 Paris, France \\ olivier.habermandorange.fr \\ ${ }^{2}$ CNAM-CEDRIC, 292 rue St Martin, 75141 Paris Cedex 03, France \\ \{romain.pellerin, eric.gressier_soudan\} @cnam.fr \\ ${ }^{3}$ Hippocad, 16 rue du Château, 77300 Fontainebleau, France \\ ugo.haberman@hippocad.com
}

\begin{abstract}
This demonstration challenges the conventional art experience. It brings together the fields of art, science, and software integration. The goal was to create a new kind of painting based on embedded technology, experimenting with a new media: paintings augmented with RFIDs. The aim was to achieve interaction between the artist's paintings and art gallery visitors.
\end{abstract}

Keywords: RFID, interactivity, art, painting, mobile phone.

The goal of the demonstration described here is to show how embedded technology could help the artist to create a better interaction between his paintings and art gallery visitors. RFID (Radio Frequency Identification) tags and mobile phone handsets came out very quickly. The artist's requirements were that the painting was free of any visual technology and that the content sent to the visitor could be changed overtime... RFID Tags were an appropriate choice to be the basis of our proposal. RFIDs are easy to use. They can be read or written through an NFC (Near Field Communications) enabled off-the-shelf inexpensive mobile handset (Nokia NFC6131). RFID Tags can be audio and text-rich information providers. They can also carry references to web sites allowing downloads of dynamic content. The communication framework that has been used in the smart painting is part of the uGASP project (gasp.ow2.org). uGASP is a middleware for ubiquitous games. This information can be altered over time by the artist, an additional dynamic and unprecedented capability. RFIDs and NFC mobile phones are a new flexible communication tool that can deliver the living voice and words of the artist thereby closing the gap between artists and the public. The technical part of the application presented here was developed in a joint project with the Hippocad Company, and the CEDRIC research lab of CNAM Paris. O. Haberman is a Painter. As an artist he has observed both at his own and other artist's exhibitions that it would be an advantage to have a direct link between the artist and the public. A way of achieving this for a wide audience with a simple service is to use the latest generation NFC Tag reader mobile phone. It promises the beginning of an enriched interaction with the artist's world.

The RFID based painting described here is provided at the demonstration. Passive tags are inserted directly inside the painting. Seven RFID Tags have been used inside the painting. Their location is indicated by symbols, elements integrated into the work 
which are easily discernible, regardless of age, culture or language of the viewer. But the RFID tags remain invisible to the public. Approaching a mobile phone from a RFID Tag (less than $2 \mathrm{~cm}$ ), the phone automatically downloads content, using audio and video programs without the need for user intervention except pushing the "ok" button. The demo shows the various point of interactivity that we have developed to date. The subject of this semi-abstract piece is Communication. The Roman numeral VIII refers to the relationship of men with their universe, the eight planets in our solar system, this allegory is represented by a photograph showing an abstract space environment. The title of the painting: "Infinite Abstraction," which refers to the invisible and infinite dimension of Communication in particular, but also of art in general, inscribed in what evokes the screen of a phone, itself element of communication, enables to send to the viewer a telephone conversation between two children, and addresses the themes of the spoken word among peoples as well as infancy, the future development of our world of Communication. The symbol $\Omega$ stamped on the piece, last letter of the Greek alphabet, used here to express the infinite possibilities of correspondence, as opposed to the start of the first communication between men, and whose graphic resonance includes a stylized bridge, invites the viewer to understand the angled triangle of the piece symbolizing a bridge between people, a physical communication link, an architectural, but also a disembodied though very real link via conversation. The number "8", chosen for the gesture of loops that can be reproduced ad infinitum, and which, overturned, brings back to the title ( $\infty$ : infinite), refers to an abstract photo in which the photographer has chosen to serve the timelessness of communication. The handwritten letter, which occupies the central place in the piece, and historical means of communication, speaks directly to the public to connect with the voice of the author the symbolism used in the creation of this painting. At last, the signature is used to display information on the identity of the piece and of the painter. Thus, this semi-abstract and symbolic piece of art offers a new reading through overt gestures towards and the interaction of many additional media that give a concrete meaning to all the elements that have made the creation of the work. In addition, each of the media has been brought to life by an artist or designer using a different mode of expression. A photographer was invited to give his analysis on his own vision of communication, children have chosen their own grid analysis of the topic and suggested a soundtrack of which they themselves have been the creators and actors, the author was given a space for an audio address to the public to attach permanently to the work the motivations which energized his thought. An additional text in the piece itself includes all the information concerning the author and the identity of the work.

The painting was shown to a range of 48 people between the ages of 12 to 56 years old, of different educational levels and socio-professional categories and with varying levels of interest in art. It allowed us to study the impact of the interaction between a painting, photos, audio messages and text on people at a painting exhibition. Before making the experiment, users have been taught for ten minutes on how the mobile phone has to be used. This approach to art/technology has enabled communication between the artist, other artists and the public. 